\title{
Justicia social... justicia territorial: ¿Un dilema sin resolver en Venezuela?
}

\author{
Social justice... land justice: an unresolved dilemma in \\ Venezuela?
}

\section{Delfina Trinca Fighera ${ }^{1}$}

\section{Resumen}

Habitualmente se endosa la responsabilidad de las injusticias sociales al sistema capitalista, argumentando que el desigual acceso a los bienes sociales tiene que ver con su naturaleza. Se podría inferir entonces que la inequidad social se habría acompañado, históricamente, de una apropiación diferencial del territorio (geodiversidad). Ante realidades sociopolíticas confrontadas con el capitalismo, ha sido común que se levanten voces clamando por justicia social y también por justicia territorial. Por ello, en su discurso es explícita la necesidad de 'equilibrar' el territorio.

La razón de reflexionar sobre este asunto se asocia con el hecho que desde la geografía, mucho más en el contexto de la globalización, pero también por la particular situación que se desarrolla desde hace más de una década en Venezuela, la preocupación por la búsqueda de la justicia social —y su correlato territorial- subyace, precisamente, al saber geográfico y a la planificación institucional de este país.

Palabras clave: geografía, desequilibrios territoriales, espacio geográfico, inequidad, justicia, ordenación, territorio.

1 Doctora en Geografía Humana de la Universidade de São Paulo, Brasil. Profesora titular del Instituto de Geografía y Conservación de Recursos Naturales, Facultad de Ciencias Forestales y Ambientales, Universidad de Los Andes, Mérida- 


\section{Abstract}

It is usually endorsed the responsible for social injustice to the capitalist system, by arguing that unequal access of population to social goods has to do with its own nature. It could be then deduced that social inequality would have historically accompanied of a differential appropriation of the territory (geodiversity). In sociopolitical realities confronted with capitalism, it has been usual to raise voices clamoring for social justice and also for territorial justice. That is why is explicit the need to 'balance' the territory.

The reason to reflect on this issue is associated with the fact that from geography, much more in the globalization context, but also by the particular situation that has being developed for over a decade in Venezuela, the concern about the search for social justice -and its territorial correlate- underlies, precisely, the geographic knowledge and the institutional planning of this country.

Keywords: geography, territorial imbalances, geographical space, inequality, justice, planning, territory. 


\section{Introducción}

El tema de la justicia, su búsqueda, forma parte del imaginario y del discurso político y social de cualquier país. ¡Y cómo no ser así! Quién puede poner en tela de juicio las innegables desigualdades sociales que muestran, en unos casos más que en otros, importantes sectores de las sociedades de los distintos países que conforman el mundo de nuestros días.

En innúmeras oportunidades se le atribuye la responsabilidad de esta realidad al sistema capitalista, argumentando que el desigual acceso de estos sectores a los bienes, servicios, educación, trabajo, salud, tiene que ver con su propia naturaleza. Casi se podría afirmar que este modo de producción, por definición, engendra riqueza y pobreza a la vez. La una de la mano de la otra.

Ante esto, parece lógico pensar que si la pobreza tiene manifestaciones materiales concretas, la población que vive en esa situación se ubicaría en aquellos lugares que, de alguna u otra forma, no son los "escogidos", "seleccionados" por los que sí tendrían la capacidad económica para decidir donde vivir. En otros términos, se podría deducir, de manera muy simple, que el desigual acceso a los bienes sociales (inequidad social) se ha acompañado, históricamente, de una apropiación diferencial del territorio, gestándose de este modo los conocidos desequilibrios territoriales.

De allí que en muchas ocasiones, frente a realidades sociopolíticas confrontadas con el capitalismo, se escuche el clamor por justicia social, pero también por justicia territorial, en el entendido que la desigualdad social y el uso diferencial que la sociedad ha hecho $-\mathrm{y}$ hace - del territorio, no se podrían explicar si no se toman en consideración los impactos generales del proceso de desarrollo capitalista. Por ello, se hace necesario 'equilibrar' (o igualar, si nos ubicamos en el plano social) el territorio, pues se parte del supuesto que los desequilibrios territoriales son consecuencia del desarrollo de este sistema socioeconómico que, por definición, es de naturaleza desigual y combinado.

Dentro del contexto de esta discusión, con este artículo se pretende presentar de manera sucinta, el tema de la justicia social y de la justicia territorial en la ciencia geográfica, sobre todo visto desde el enfoque de la geografía radical. A continuación se analiza el tema, centrado en la noción de equilibrio versus desequilibrio territorial, a la luz de la realidad sociogeográfica venezolana de los últimos cincuenta años (período democrático).

\section{Algunas aproximaciones concep- tuales necesarias}

La noción de justicia social, en tanto que concepto normativo, aparece a mediados del siglo XIX, asociada con la necesidad de buscar y alcanzar un reparto equitativo de los bienes sociales. En esa búsqueda, el Estado emerge, sin duda, como un actor de primera línea, puesto que se parte del supuesto que su accionar es fundamental para encarar 
las diferencias que generan las sociedades, con miras, precisamente, a minimizarlas.

Desde esta perspectiva, ¿qué es justo y qué no? Difícil responder a esta interrogante y, más aun, precisar una definición. La 'justicia' va a depender, fundamentalmente, de los valores que tenga una sociedad, pero también de las distintas formas de concebir la vida de cada persona. Sin embargo, se puede afirmar que la idea de justicia se relaciona con lo que 'debe hacerse' de acuerdo con lo equitativo, razonable (darle a cada quien lo que le pertenece o concierne), o lo dictado por el derecho. Pero, ¿quién es el responsable de generar un lugar justo y equitativo? ¿Qué papel juega el Estado en esa búsqueda? y, ¿todos estarían de acuerdo con cuál es el rol que debe asumir el Estado para encarar las desigualdades sociales?

Existen distintas corrientes de pensamiento con propuestas diversas para enfrentar este problema. En general, los defensores del liberalismo afirman que el Estado debe apuntar a garantizar la generación de oportunidades para todos, pero incentivando y protegiendo las iniciativas privadas. Por el contrario, las corrientes socialistas se centran en la intervención estatal para alcanzar la justicia social. Tanto unos como otros, tienen como utopía satisfacer por 'igual' el acceso a los bienes y servicios sociales (alimentos, vivienda, salud, educación), siendo que esto solo se podría lograr por medio de la intervención del Estado, que debe regular las relaciones sociales vía leyes y reglamentos, y con progra- mas sociales que, de manera progresiva, tiendan a superar las diferencias existentes entre los distintos sectores de una sociedad (Interficto, 2010).

En fin, sobre este tema es mucho lo que se ha escrito y se argumenta, pero nunca faltará quien sostenga que sus necesidades no han sido satisfechas, mientras que otros dirán, por el contrario, sentirse afectados cuando los gobiernos instrumenten, por dar un ejemplo, programas sociales sustentados en pechar con más impuestos a los que más tienen. Difícil compensar lo que cada sector social reclama como suyo por derecho.

En geografía, el tema de la justicia social es relativamente reciente. Su emergencia como preocupación entre la comunidad geográfica escasamente llega al medio siglo. Se asocia y mucho, con el surgimiento de la llamada corriente radical en el pensamiento de esta disciplina. Los sucesos que marcaron los años sesenta y setenta del pasado siglo XX (Mayo francés, guerra de Vietnam, asesinato de Martin Luther King, el movimiento hippie, entre los más destacados), impulsaron a más de un pensador de la geografía a mirar hacia este tema, el cual, aun cuando su campo de acción está mucho más asociado con las ciencias sociales y humanas, lo abordaron desde la perspectiva territorial (o espacial, en algunos casos).

Harvey, en el libro Urbanismo y desigualdad social (1977), cuya primera edición en inglés sale a luz pública en 1973, bajo el título de Social Justice and de City, discute de manera explícita este 
asunto de la justicia social. Si bien es bastante cuidadoso en sus argumentaciones, no deja de afirmar que es necesario "construir una teoría normativa sobre la asignación espacial o territorial basada en los principios de la justicia social" (1977, p. 98); sugiere que para ello se debería dejar de lado el concepto de eficiencia, basado en la oferta y demanda, como principio regulador y minimizador de las diferencias espaciales, y trabajar mucho más en la idea de la distribución. En otros términos, la noción de justicia social es el eslabón que le facilita proponer una justicia distributiva territorial (justicia social territorial). El ingreso es lo que se distribuye en el territorio (acceso a los recursos escasos de la sociedad) y se puede repartir tanto entre individuos como entre unidades territoriales.

Años más tarde, Harvey (1992, 1996), se concentra mucho más en indagar cómo funciona espacialmente el capitalismo, desarrollando la tesis del materialismo histórico geográfico. Da este 'giro' porque se encuentra con algunos problemas para explicar los mecanismos que producían las distribuciones territoriales injustas. Poco después retoma este asunto, pero haciendo énfasis en las dificultades relativas al análisis de la justicia social; entre otras cosas, destaca lo difícil de conciliar los ideales de derechos e igualdad universal con las múltiples diferencias económicas, sociales, étnicas, culturales de los distintos lugares que conforman el mundo (universal/ particular). También resalta la importancia de retomar los conceptos básicos de la ciencia geográfica como punto de partida para sentar las bases del materialismo geográfico-histórico.

Además de Harvey, otros autores también se preocupan por este dilema, pero más que partir de una discusión de la justicia social y su correlato territorial, centran ${ }^{2}$ su análisis en el estudio de las estructuras sociales y sus relaciones con las formas espaciales, señalando que la explicación estaría en los modos de acumulación de capital, puesto que la organización (diferencial) del espacio sería resultado de la dinámica social. De hecho, dan por sentado que las leyes del capital son las que ayudan a explicar su expansión espacial y su concentración en algunos lugares (y no en otros), con lo cual las diferencias espaciales no se disocian de las diferencias sociales: las últimas explicando a las primeras.

Una de las principales trabas con la que tropiezan estas reflexiones, es que la teoría marxista clásica no desarrolló una dimensión espacial explícita; a pesar de ello, para muchos geógrafos que asumieron esta teoría, colocar lo social y económico como fuente primaria de explicación de las desigualdades territoriales, era coherente, dada la naturaleza desigual y combinada de manifestarse del modo de producción capitalista. De esta manera, en muchos casos, el

2 Entre muchos están: Lefebvre (1974), Peet (1977), Smith (1988), Smith (1980), Slater (1973), Soja (1993), Santos (1975), Moraes y Messias da Costa (1984), Rawls (1971), Reynaud (1981). Más recientemente, la revista Annales de Géographie dedicó un número temático $(2009,665-666$,$) a$ tratar este asunto; así mismo, Fournier (2011) discute sobre esto, tomando como ejemplo el caso de la Venezuela actual. También Soja (2010) continuó trabajando en esta línea, así como Bosque et al., (2004). 
análisis de la realidad espacial tenía su explicación dada a priori.

En las últimas décadas, este tema de la justicia social vuelve a emerger en el campo de la geografía humana. Entre otras, retorna al escenario la vieja idea de la eficiencia 'espacial', pero ahora la búsqueda por una 'adecuada' distribución de infraestructuras generadoras de bienestar social, debería centrarse en minimizar las distancias entre la oferta de un servicio y su demanda; por ejemplo, para instalaciones 'deseables' como hospitales o escuelas, se buscaría disminuir las distancias entre ellas y las personas que deben usar el servicio (acceso por parte de la población), pero para otras no 'deseables' (las que potencialmente pueden producir daños y peligros en su entorno), se buscaría lo contrario: incrementar esas distancias $^{3}$, pero siempre con el objetivo de que estas distancias no fuesen muy desiguales; así se evitaría que unas personas pudiesen resultar más afectadas que otras.

Este concepto de justicia espacial (eficiencia espacial) se acompaña de otro: el de justicia ambiental. La justicia es-

3 Bosque, Díaz, C. y Díaz, M. (2004), señalan que: "Complementario al concepto de eficiencia espacial es el de justicia espacial. (...) se trata de que las instalaciones no estén desigualmente repartidas sobre el espacio, de modo que no existen diferencias, 0 al menos éstas no sean excesivamente grandes, en el acceso de la población a estas instalaciones. Este principio es muy importante en el caso de instalaciones financiadas con fondos públicos. En el caso de equipamientos no deseables nuevamente se trata de que las distancias entre la población y los equipamientos no sean demasiado desiguales, de modo que unas personas se vean muy afectadas y otras no lo estén en absoluto". pacial se centraría en la búsqueda de la óptima distribución espacial de los equipamientos 'deseables', mientras que la ambiental representaría la adecuada distribución de las infraestructuras 'no deseables'. Aun cuando esta aproximación es más utilitaria que otra cosa, en realidad es bastante reduccionista, pues restringe el problema a las distancias (accesibilidad espacial), dejando de lado lo que sería una de las ideas centrales de la justicia social: reducir el desigual acceso de las personas a los bienes sociales.

Soja (2010) es otro geógrafo que ha incursionado en esta discusión relativa a la justicia social y territorial. Si bien hace ya un buen tiempo, en referencia a la dialéctica socio-territorial, señalaba que las relaciones sociales forman el territorio y que las territoriales forman a su vez a las sociales y de clase - dando así un paso adelante con relación a las posturas 'tradicionales' en el contexto de la geografía radical-, hoy en día, con la publicación en el 2010 de su libro Seeking spatial justice (citado por Link, 2011), elabora el concepto de justicia espacial en el marco de esa relación dialéctica socioterritorial. La justicia, vista desde cualquier ángulo, tiene implicaciones geográficas y expresión espacial, por lo que el espacio sería algo 'más' que una mera característica externa del mundo social. Así, a la vez que la sociedad produce espacialidades injustas, el espacio también es fuente de injusticias $^{4}$. El giro espacial propuesto por En este sentido, Soja señala que la creación de injusticias
espaciales es de naturaleza multiescalar. Hace referencia 
este autor consiste, "precisamente, en asumir que no hay ninguna razón para entender que nuestra existencia social e histórica es más importante en cualquier sentido que nuestra existencia espacial" (citado por Link, 2011, p. 176). En su opinión, si los procesos sociales no se espacializan de manera uniforme, la desigualdad en la distribución es la característica más obvia de la injusticia espacial.

Santos (1996) también da un salto cualitativo al afirmar que el espacio es algo más que un producto social, puesto que asumido de esta manera, facilita descuidar, por no decir dejar de lado, la influencia del espacio 'construido' sobre la propia sociedad. Si bien este autor no discute de manera explícita el problema de la justicia social/territorial (o espacial), sus planteamientos teóricos transitan una ruta similar a la de Soja, en cuanto a la importancia que le cabe al espacio en la explicación de lo social.

Cuando Santos sostiene que toda sociedad tiene una manera de usar su territorio $^{5}$ y su tiempo, y que en este proceso redefine, continuamente, su materialidad, no hace más que reafirmar que la configuración territorial de un lugar,

a escalas globales (geografías exógenas), regionales (mesogeografías) y locales (geografías endógenas). En cada una de ellas, se dan situaciones de injusticia espacial; por ejemplo, en las 'geografías endógenas' resalta la discriminación geográfica que resulta de leyes, normas y toda clase de reglamentaciones (citado por Link, 2011).

5 El término territorio, lato sensu, se utiliza para referirse a aquellas porciones de la superficie de la tierra, sobre las que el hombre social, históricamente, ha tomado posesión; en consecuencia, están sujetas a relaciones de poder; en un sentido más estricto, es la denominación política para el espacio. región o país, solo tiene sentido y significado para esa sociedad a través de sus acciones (Santos, 1996), resaltando que estas acciones definen funciones específicas, concretas para los objetos que ha construido y que se incorporan, de manera constitutiva, al territorio en referencia. De allí que el territorio esté sujeto a múltiples y sucesivas modificaciones en función del uso que la sociedad, a lo largo de la historia, hace del mismo. Por ello, la comprensión del territorio usado solo tendría sentido en relación con las acciones que emanan de esa sociedad.

Desde esta perspectiva, la sociedad no sería solamente el origen de las acciones sino que de estas participaría el territorio usado, resultando difícil aproximarse a la comprensión del espacio (territorio usado), independientemente del tiempo histórico; de la misma forma, resultaría bastante engorroso imaginar una sociedad actuando sin espacio, o fuera de él. Empíricamente, de lo que se trata es de periodizar el tiempo y diferenciar el espacio, pero simultáneamente; es decir, analizar la espacialidad de los procesos históricos, lo que significa seguirle el curso a la sociedad en tiempo y espacio. Ello obliga a un decidido esfuerzo mental para poder pensar, a un tiempo, los objetos (la materialidad), las acciones (la sociedad), y sus mutuos condicionamientos, valga decir, las relaciones (Rojas \& Gómez, 2010).

En síntesis, la historia nos dice que la forma como los seres humanos, organizados socialmente, han tomado posesión 
de la base material que les da sustento ha sido - y continua siendo- desigual, y este uso diferencial reafirma que el proceso de ocupación no se realiza al margen de las intenciones que guían u orientan a esos grupos. Pero también nos dice que esas intenciones se cristalizan en objetos cuya existencia tiene que ver con las necesidades propias de cada momento histórico, objetos que, al acumularse de manera desigual en los territorios (Santos, 1996), le incorporan valores diferenciales que terminan, de manera progresiva, por imponer realidades que deben ser, necesariamente, consideradas a la hora de tomar decisiones que signifiquen cambios en los usos de esos territorios. En este contexto, el puzzle de lugares que conforman el espacio geográfico es un resultado histórico de la particular combinación de condiciones naturales y sociales propias de cada lugar, a las que se le deben agregar, en nuestro tiempo, las variadas y diferentes combinaciones entre lo local y lo global en una sucesión de múltiples escalas.

Compartimos con Soja que los procesos sociales no se espacializan uniformemente. Nuestras acciones y actividades tienden a focalizarse alrededor de un centro o aglomeración, lo que traerá como consecuencia una distribución desigual "de ventajas y desventajas, dependiendo de la localización y accesibilidad respecto a dicho centro (...). La vida humana no está configurada por desigualdades geográficas, sino que esta configuración juega un rol en producirlas y reproducirla." (Soja, citado por Link, 2011, p. 176).

\section{Justicia espacial: ¿contraparte necesaria a la justicia social en Ve- nezuela?}

A partir de los años 60 del pasado siglo XX, el Estado venezolano define como uno de sus objetivos más importantes implementar políticas que se orienten a minimizar los desequilibrios territoriales; para ello parte de la idea de que se debe diversificar la economía y, paralelamente, favorecer un desarrollo que propenda a la justica social ${ }^{6}$ (equitativa distribución de la riqueza, disminución progresiva de la pobreza), en un contexto en el que se privilegie el respeto al ambiente. Si la búsqueda de un desarrollo territorial 'equilibrado' fue una de las preocupaciones de los distintos gobiernos del "período democrático", pareciera que a lo largo de la historia, las acciones de la sociedad venezolana habrían propiciado una organización no 'equilibrada' de su espacio. Ante estos planteamientos nos preguntamos: ¿Por qué desde las distintas administraciones centrales de la Venezuela del presente se parte del hecho de que el territorio no está 'equilibrado'? ¿Por qué se insiste en la actual administra-

6 Si bien los distintos gobiernos del llamado período democrático (ver nota 6) no se refieren de manera explícita a la justicia social (con la excepción de los gobiernos del presidente Chávez), los principios que esta noción encierra, si están recogidos en las distintas políticas sociales, económicas y territoriales diseñadas e implementadas por cada uno de ellos.

7 Esta expresión se refiere al período que se inicia con la administración del presidente Rómulo Betancourt (19591964), después de la caída de la dictadura del general Marcos Pérez Jiménez el 23 de enero de 1958, y que continúa hasta el presente, con el gobierno del presidente Chávez, electo por cuarta vez para una nuevo período presidencial en las elecciones del 7 de octubre de 2012. 
ción del presidente Chávez en esta idea del no equilibrio?

\subsection{Algunos antecedentes del porqué de la actual organización del espacio venezolano}

Aproximándonos un poco al pasado territorial de Venezuela, constatamos que desde los tiempos prehispánicos, los pobladores originarios de lo que hoy es la República Bolivariana de Venezuela, se emplazaban en lo que también hoy son las áreas de mayor concentración de población y de actividades de todo tipo: nos referimos en particular al arco montañoso andino-costero (Figura 1).

La colonización española comienza y se consolida en un territorio ya ocupado y 'usado', con una configuración que denota diferentes grados de complejidad en el desarrollo y uso de técnicas por parte de los grupos humanos allí asentados. Los españoles se encuentran así con áreas pobladas en las regiones: centro-costera, andina, noreste y noroeste,

Figura 1. Hidrografía y ciudades más importantes

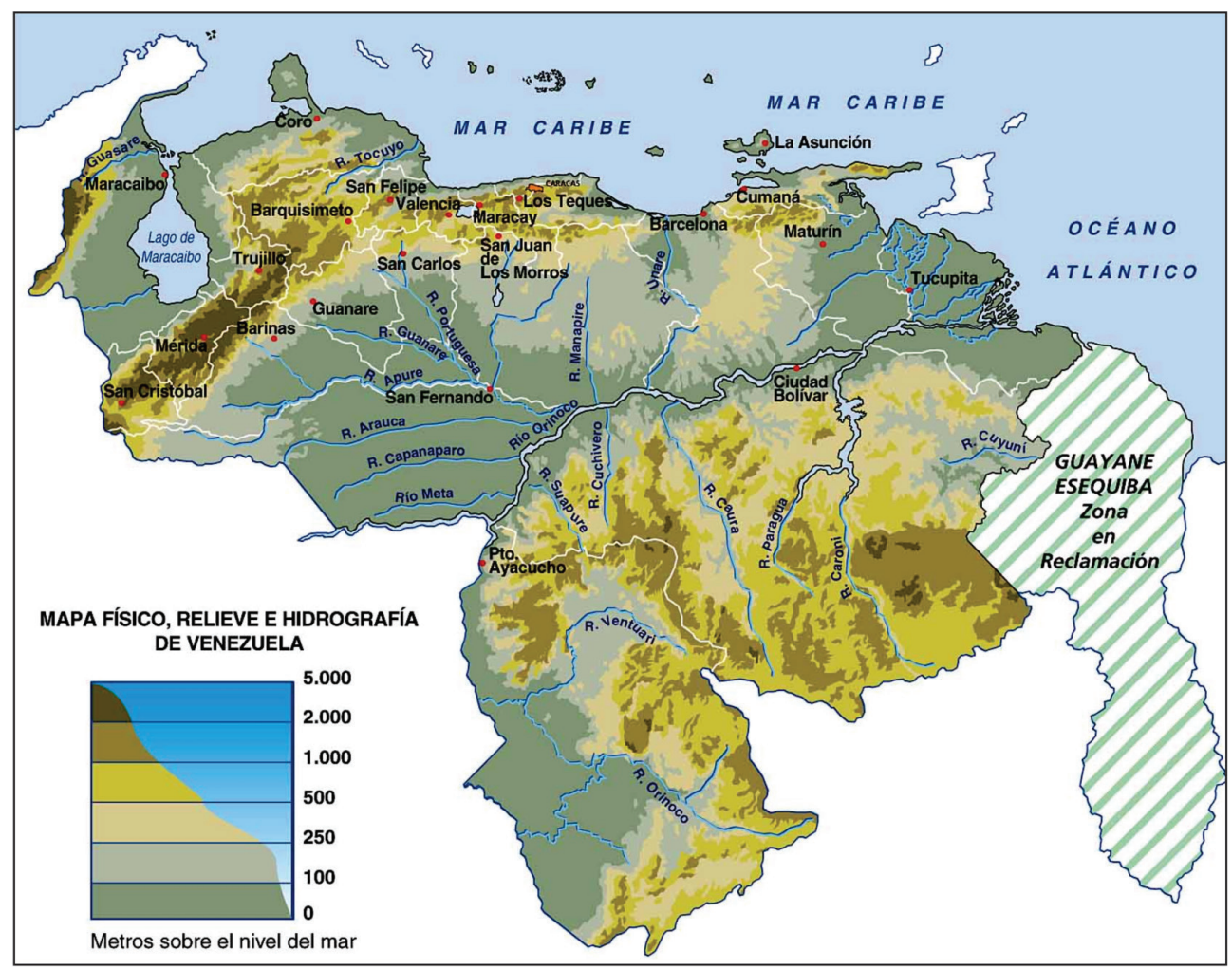

Fuente: Mapas de relieve de Venezuela, 2012 
precisamente aquellas donde se localizaban las tierras más fértiles, ubicadas en el mencionado arco montañoso. También había presencia humana en el resto del territorio venezolano, pero con pocas excepciones aún tenía como base la 'trashumancia', por lo tanto con una impronta en la que lo 'natural' aún era lo determinante.

El control español se centra, en consecuencia, en ese arco andino-costero montañoso, sobre todo en el área centro-norte. Allí desarrolla una economía agroexportadora (siglo XVIII), sustentada en el cultivo del cacao (también había presencia de caña de azúcar, algodón, tabaco, café). Por su valía económica, esta región se constituye en asiento del poder colonial y de los principales puertos de la época: Puerto Cabello y La Guaira. Para finales del siglo XVIII contaba con aproximadamente 90.000 habitantes (Rojas \& Pulido, 2009). En el noroeste, la ciudad de Maracaibo aprovecha su posición geográfica en la cuenca del lago del mismo nombre, y desarrolla una importante red comercial en su hinterland andino.

El resto del país, con excepción del citado arco montañoso andino-costero y sus piedemontes, se mantiene casi despoblado, con alguna presencia humana que escasamente podría formar aldeas, aisladas y no incorporadas al resto del país (Trinca, 2005). De esta forma, el espacio de la Venezuela colonial se va formando diferencialmente, generando una organización que se sustenta en la que ya existía para el momento de la llegada del conquistador español.
A inicios del siglo XIX, Venezuela, al igual que la mayoría de las colonias españolas de América, se ve envuelta en un serio conflicto armado que tiene como sus detonantes más significativos la revolución de 1810 y la declaración de Independencia, un año más tarde (5 de julio, 1811). A esta crítica situación se le añade la destrucción de su capital por el conocido terremoto de Caracas (1812), sucesivas crisis económicas y un sinnúmero de guerras civiles. Un resultado casi inmediato de esta realidad fue el derrumbe de la agricultura de plantación de base esclavista y de la economía ganadera. Así, el café, cultivado ya con fines comerciales desde finales del siglo XVIII, desplaza, para mediados del XIX, al cacao como principal producto de exportación.

En el marco de las transformaciones que experimenta la economía internacional, sobre todo a partir de mediados del siglo XIX, los distintos gobiernos de los nacientes Estados Unidos de Venezuela, en particular los del general Antonio Guzmán Blanco (1870-77; 1880-84 y 1886-87), concentran sus esfuerzos en construir un "estado nacional burgués centralizado,..." (Chaves, 1992, p. 132).

Para cumplir con este propósito, aun cuando la situación fiscal del Estado de aquel momento no era la mejor, el gobierno de Guzmán Blanco impulsa la construcción de obras de infraestructura. Es una incipiente red ferroviaria ${ }^{8}$ la

8 Entre 1883 y 1892 se construyeron un poco más de 1.052 $\mathrm{km}$ de líneas férreas. 
que contribuye para que el territorio venezolano comience a mostrar una embrionaria articulación, solo que la misma se concentra en los lugares donde se cultivaban los principales productos de exportación, valga decir café y cacao (arco montañoso andino costero).

A pesar de estos focalizados esfuerzos, la Venezuela que recibe al siglo $\mathrm{XX}$ sigue siendo un país muy poco articulado, escasamente poblado, rural y pobre ${ }^{9}$. Caracas, a pesar del crecimiento de algunas regiones y ciudades vinculadas directamente con la economía agroexportadora, refuerza en este período su primacía no solo por ser la sede del poder político y administrativo del país, sino también porque se constituye en el principal centro (y por extensión su área de influencia) beneficiario de la riqueza generada por la economía agroexportadora.

No sorprende entonces que para 1920 la región centro-norte-costera concentrase el 45,2 \% del total de la población del país residente en centros poblados mayores de 10.000 habitantes; Caracas con sus 92.212 habitantes y Valencia con 29.466, absorbían cerca del $90 \%$ de ese porcentaje. A esto hay que agregarle los puertos de La Guaira y Puerto Cabello que, en la práctica, se constituyeron en una ex-

9 Para 1920, Venezuela rondaba los tres millones de habitantes, de los cuales solo el $27,3 \%$ vivía en centros poblados con un poco más de 2.500 habitantes. El promedio del producto nacional por habitante apenas llegaba a los 147 dólares, y la esperanza de vida a duras penas alcanzaba los 34 años (Baptista, 1985). tensión 'natural' de estas dos ciudades (Estaba, 1985).

También para 1920, Maracaibo, en el extremo noroeste, es la segunda ciudad del país con 46.706 habitantes. Nada inusual si pensamos que es el centro mercantil por excelencia de la región productora de café más importante de la Venezuela de comienzos de siglo: los Andes. Entre 1873 y 1920, Maracaibo y las ciudades andinas de San Cristóbal (capital del estado Táchira) con 21.385, y Mérida (capital del estado homónimo) con 14.082, concentraban el 27,5 \% del total de la población del país viviendo en ciudades de 10.000 o más habitantes (Estaba, 1985). Esto quiere decir que un poco más del $70 \%$ del total de la población venezolana para finales del siglo XIX y primeras décadas del XX, se localizaba en el área centro-nortecostera, en el noroeste (Maracaibo), y en los Andes, es decir, esencialmente, en el arco montañoso andino-costero.

El porcentaje restante se lo repartían de manera desigual la región noreste, los Llanos y Guayana. De estas, la balanza se inclina a favor de la noreste, sobre todo por la presencia de la ciudad-puerto de Carúpano, la cual llegó a contabilizar una población estimada para 1920, de unos 20.000 habitantes. Su cercanía a zonas productoras de cacao y café, ayudan a entender este crecimiento. Con los Llanos y Guayana, por el contrario, se observa una clara tendencia hacia el decrecimiento de su población, principalmente entre 1891 y 1920, asociada con la decadencia de 
la economía ganadera, el aislamiento en el que se mantiene el sur del país y la importancia que comienzan a adquirir ciudades como San Cristóbal, Mérida y algunas otras de la región centro-norte-costera (Estaba, 1985).

Por lo señalado hasta ahora, se constata como la maraña de fuerzas y factores de todo tipo (internos y externos), se combinan en cada momento histórico para cambiar, reforzando o no, lo que nuestro pasado territorial nos legó. Es esta particular combinación de actores y sus intenciones plasmadas en objetos concretos, la que contribuye a determinar que sea hoy por hoy la región centro-norte-costera la que capitaliza la mayor concentración demográfica, económica y de intercambios de todo el país.

\subsection{Petróleo: ¿cambios en la orga- nización espacial?}

Aun cuando en Venezuela se observan algunos intentos por explotar comercialmente el petróleo a finales del siglo XIX, por medio de la Compañía Minera Petrolia del Táchira (al suroeste del país), que lo extrae con la finalidad de producir queroseno para el consumo local, no fue sino hasta 1912 cuando formalmente se inicia la exploración para buscar y explotar, con fines comerciales, este combustible mineral. Es tan importante esta nueva actividad, que una de las primeras señales de lo que va a significar para la futura economía del país se observa en la composición de las exportaciones: en menos de veinte años, Venezuela deja de ser agrario para transformase en petrolero ${ }^{10}$.

Para mediados de la década del veinte, la nueva actividad económica ya se hace sentir de manera importante en la estructura económica y social de la Venezuela rural de comienzos del siglo XX, al facilitar, de manera expedita, un sostenido y progresivo incremento de la capacidad de demanda, tanto interna como externa, que favoreció, a medio plazo, las formas de apropiación financieras y consumistas sobre las productivas. Estas formas de apropiación intensifican la dinámica de los servicios y la libre y barata importación, la que se torna entonces mucho más rápida que la que requiere el aparato productivo interno.

Un impresionante traslado de población rural hacia las áreas donde la actividad petrolera se realizaba de forma directa (occidente y oriente del país), fue un primer resultado de esta nueva situación ${ }^{11}$. De igual manera, las principales ciudades donde tra-

10 Para 1920, las exportaciones petroleras apenas representaban el 1,7\% del total, mientras que las de café, cacao y otros rubros agrícolas y mineros aportaban el 98,3 \% (sólo el café y cacao contribuían con el 58,1\%); quince años más tarde (1935), el petróleo llegaba al 86,1\%, quedando reducidas las del sector agrícola y minero al 13,9\%. Ya para 1928, Venezuela era el primer exportador de petróleo del mundo (Trinca, 2008).

11 Además de todos los 'nuevos' objetos que tienen que ver con la explotación petrolera, también surgen casi que de la nada, las llamadas ciudades petroleras que nacen, muchas de ellas, a la sombra de los campos petroleros convirtiendo pequeñas aldeas aisladas y sin ninguna importancia, en activos centros que cambian radicalmente el paisaje de los lugares donde se localizan. 
dicionalmente el poder económico y político se había asentado (Caracas y Maracaibo en particular), también reciben importantes contingentes de población, al ser beneficiarias de fuertes inversiones efectuadas por el Estado. Por tanto, a partir de la década del treinta, el petróleo se constituye en el factor fundamental para que el Estado consolide su incipiente naturaleza centralizadora y concentradora; pero también para que la fuerza de trabajo comience su transformación: a su sombra se gesta la clase obrera, se expande la clase media y se forma y fortalece el empresariado nacional (Trinca, 2005).

Los efectos de la abundancia de divisas se hacen sentir con mayor intensidad desde mediados del siglo pasado, con la política de sustitución de importaciones y la necesaria construcción de las bases materiales para el desarrollo nacional: modernización del campo, industrialización, red de carreteras ${ }^{12}$; todo este dinamismo se acompañó del fortalecimiento del sistema de ciudades, pero también de la ampliación de los cinturones de pobreza en las principales ciudades, generándose un patrón urbano-concentrado con una dominante: las áreas metropolitanas del centro-norte, y otras aglomeracio-

12 A fines de los años cuarenta del pasado siglo XX, el Estado diseña el Plan Preliminar de Vialidad; allí se estimaba construir veinte carreteras principales (troncales), con sus ramales de enlace: eran más de $17.000 \mathrm{~km}$. Para inicios del XXI existen más de $100.000 \mathrm{~km}$ de carreteras entre pavimentadas y no pavimentadas, una realidad bien distinta a la que mostraba el país antes de la Segunda Guerra Mundial, cuando solo había un aproximado de 6.000 km (Trinca, 2005). nes de menor jerarquía en el resto del país.

Es importante señalar que la lógica del modelo de desarrollo adoptado fue la de consumir primero y producir después. Esta lógica se sustentó, entre otras cosas, en la decisión política de mantener sobrevaluada la moneda nacional (bolívar), pues esto le permitía al Estado mantener un flujo constante de bolívares, pero una cantidad substancialmente mayor de dólares. Esta decisión también facilitó un aumento impresionante del ingreso nacional, el cual se transfiere a la sociedad venezolana que, de esta forma, adquiere el hábito de consumir todo tipo de bienes importados. Así se estructura una economía abierta a las importaciones, pero cerrada a las exportaciones no petroleras, ya que la sobrevaluación del bolívar no favorecía que parte de la producción nacional de origen no petrolero pudiese colocarse, de manera competitiva, en el mercado externo.

Esta situación contribuye para que los centros que históricamente habían detentado el poder político terminen por transformarse en aquellos donde también se localiza la dinámica económica, dándose de esta manera esa 'sobreposición' territorial de lo político y lo económico, alrededor de las relaciones sociales que surgen a la sombra de la distribución y consumo de la renta petrolera. No es extraño entonces que en esa gran región del centro-norte del país, se concentre el mayor número de industrias, servi- 
cios, ingresos y personas ${ }^{13}$.

\subsection{Políticas territoriales y la búsque- da del 'equilibrio territorial'}

Es en este contexto que las políticas territoriales que se plantean en Venezuela, a partir del período democrático, y recogidas en los diferentes planes de la Nación, incorporan el tema de los 'desequilibrios territoriales'. En efecto, en ellos se puede observar como las estrategias en materia territorial debían centrarse en:

Desconcentrar el área metropolitana de Caracas, promover metrópolis regionales y polos subregionales (...), conectar los territorios a través de ejes de desarrollo, incentivar el desarrollo de las áreas potencialmente agropecuarias y fomentar la conservación de las áreas protegidas. Los proyectos del Eje Apure-Orinoco, la Conquista del Sur y la Áreas Rurales de Desarrollo Integrado, (...), se inscriben en la concepción de los necesarios equilibrios territoriales de la nación, que se postulaban desde los años sesenta (Salas-Bourgoin, Pulido, Rojas \& Páez, 2011, p. 35).

Por ello, la intención de 'equilibrar el territorio', aprovechando los recursos, pero acompañado de la necesaria con-

\footnotetext{
13 Por ejemplo, para 1998, las regiones Capital y Central, con tan solo el 2,3\% del territorio nacional, concentraban, entre otras cosas, el $36,3 \%$ del total de la población, el $60,5 \%$ del total del empleo de la industria manufacturera y más del $70 \%$ de los establecimientos industriales (Venezuela Analítica, 2001).
}

servación del ambiente, ha sido desde ese momento, uno de los objetivos centrales de la política de ordenación del territorio que se diseña e implementa en Venezuela, ya desde los años sesenta del pasado siglo XX. Si bien esta política se plasma en la ley correspondiente ${ }^{14}$, aprobada en 1983, numerosos instrumentos legales formulados y sancionados por el poder legislativo desde entonces (o por vía habilitante), dan cuenta de estas intenciones ${ }^{15}$.

Las décadas finales del siglo $\mathrm{XX}$, período turbulento para Venezuela, conocen del interés del Estado por revisar conceptualmente las propuestas de ordenación del territorio, aprovechando que aún estaba pendiente, entre otras, la aprobación del Plan Nacional de Ordenación del Territorio (PNOT), el cual finalmente fue sancionado en 1998. A pesar de la revisión, este plan continúa cobijando los principios de sostenibilidad y equidad.

14 En la Ley Orgánica para la Ordenación del Territorio, la ordenación del territorio es entendida como "la regulación y promoción de la localización de los asentamientos humanos, de las actividades económicas y sociales de la población, así como el desarrollo físico-espacial a fin de lograr una armonía entre el mayor bienestar de la población, la optimización de la explotación del uso de los recursos naturales y la protección y valoración del medio ambiente, como objetivos del desarrollo integral." (resaltado nuestro), (República de Venezuela, 1983, art.3).

15 A manera de ejemplo podemos citar la Ley Orgánica del Ambiente (1976), en la que ya se menciona a la ordenación, en el sentido de que el territorio debía ordenarse en función de dar el 'mejor' uso a los espacios "de acuerdo a sus capacidades, condiciones específicas y limitaciones ecológicas." Estas ideas aparecen en el V Plan de la Nación (1974-1978), y especialmente recogidas en los documentos de creación del Ministerio del Ambiente y de los Recursos Naturales Renovables (MARNR) en 1977. 
Con la llegada de una nueva administración gubernamental, en 1999, el Estado venezolano no implementa políticas territoriales al margen de estos principios. Abandonando el recién aprobado plan de 1998, diseña el Plan Nacional de Desarrollo Regional 2001-2007 (República Bolivariana de Venezuela, 2001), en el que se expone, de manera absolutamente explícita, que el proceso de ocupación territorial es la causa fundamental de los problemas socioeconómicos del país: "Esta desequilibrada ocupación del territorio nacional creó graves problemas sociales y ambientales en sus principales ciudades y restó dinamismo a la provincia" (República Bolivariana de Venezuela, 2001, p. 7). También se le atribuyó a los desequilibrios territoriales el estancamiento económico, las migraciones, la marginalidad, la pobreza y el deterioro ambiental, lo cual poco contribuyó al desarrollo general del país.

En consecuencia, este plan se hace eco de lo propuesto en el primer programa de gobierno del presidente Chávez, en el cual se sostenía que era necesario revertir este proceso, por cuanto lo hecho, sobre todo en los últimos cuarenta años, en materia de desarrollo territorial estuvo errado, ya que, en vez de propiciar una efectiva desconcentración y descentralización de actividades y personas, profundizó la 'concentración del desarrollo nacional, (...) en una pequeña porción del territorio, dejando el resto (...) prácticamente desatendido (...),' (Venezuela Analítica, 2001), por lo que era imperativo impulsar un proceso de equili- brio territorial que propendiese a distribuir más equitativamente tanto a la población como a las actividades económicas.

Con el fin de promover esa ocupación 'equilibrada' del territorio, el Plan presentó una estrategia de descentralización-desconcentrada basada en la conformación de ejes ${ }^{16}$ y áreas de desarrollo: dos ejes de desarrollo territorial con orientación norte-sur en los extremos del país y un eje transversal o intermedio con orientación este-oeste (Figura 2). El eje occidental incluye la cordillera andina y la cuenca del lago de Maracaibo (aproximadamente $10 \%$ del territorio nacional y $23 \%$ de la población del país para el 2001). El eje oriental integra los territorios de la fachada atlántica, desde el estado Nueva Esparta hasta el oriente de Guayana (21 $\%$ del territorio y $1 \%$ de la población). Por último, el eje transversal o ApureOrinoco, constituido como 'proyecto bandera', abarca un territorio de más de $300.000 \mathrm{kms}^{2}$ (cubre el $30 \%$ del territorio, pero solo el $12 \%$ de la población), (Rojas \& Pulido, 2009).

Con esta estrategia se buscaba 'equilibrar' la distribución territorial del ingreso, de acuerdo con el aprovechamiento de las potencialidades regionales, así como de las actividades

16 En el Proyecto Nacional Simón Bolívar. Primer Plan Socialista 2007-2013, además de los tres ejes propuestos, se añaden los ejes: Norte-Llanero y Norte-Costero, siendo el Norte-Llanero "...la bisagra estratégica del desarrollo regional (...) para alcanzar mayores niveles de satisfacción y justicia social..." (República Bolivariana de Venezuela, 2007). En este plan, la idea del equilibrio territorial se mantiene. 
Figura 2. Ejes de desarrollo regional propuestos

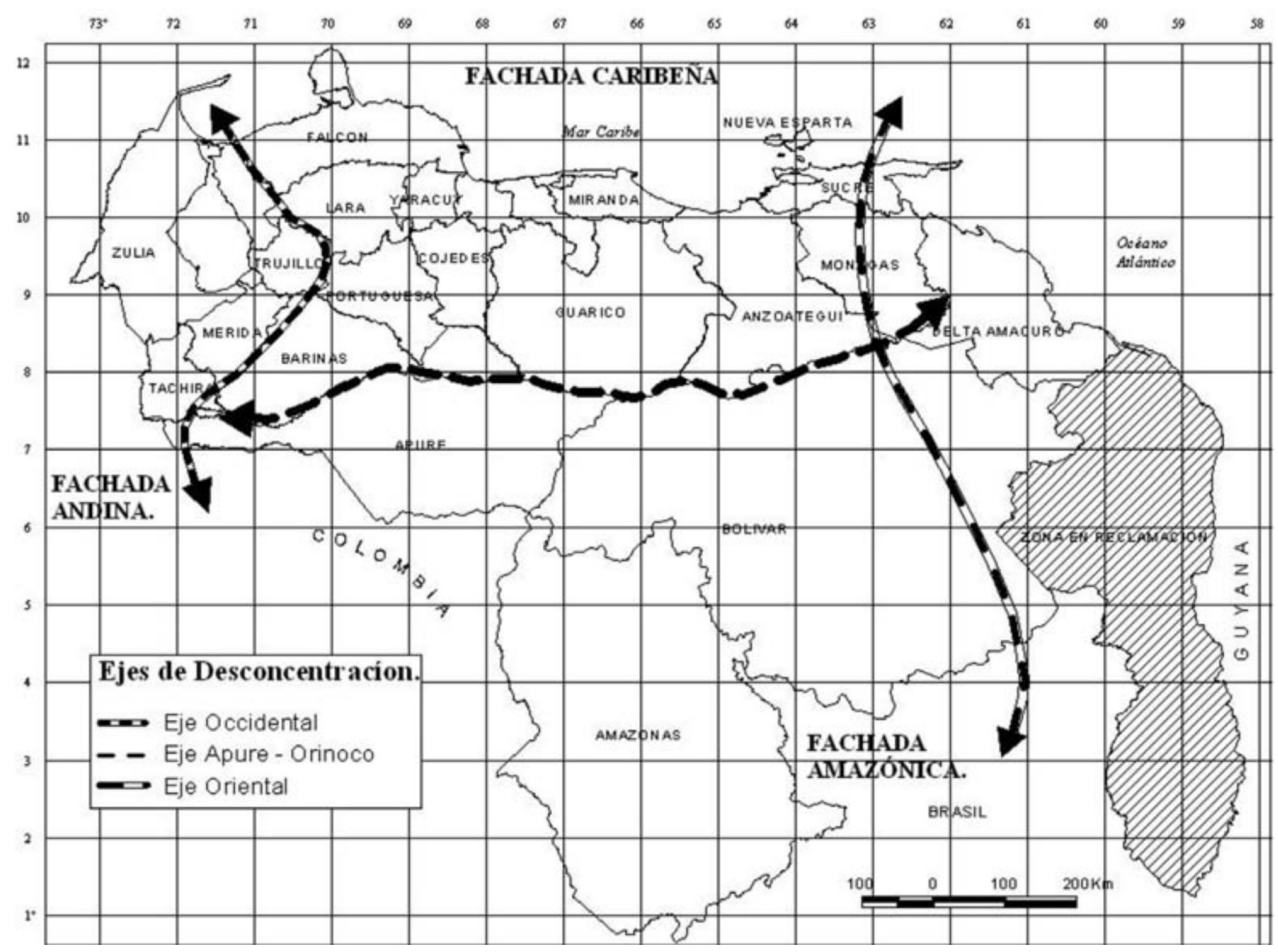

Fuente: Rojas y Pulido (2009, p. 12)

productivas y la ocupación 'racional' y eficiente del territorio. Sin embargo, estas 'intenciones' no se acompañaron de programas generales para los ejes, que estuviesen articulados territorialmente a un proyecto nación, sino que se optó por orientar inversiones focalizadas hacia algunas áreas empíricamente seleccionadas como las Zonas Especiales de Desarrollo Económico Sustentable (ZEDES), Núcleos de Desarrollo Endógeno (NUDES), Sistemas de Asociaciones Rurales Auto-Organizadas (SARAOS), entre otros. Estas últimas áreas de desarrollo, no obstante, no eran innovaciones puesto que ya habían sido propuestas en la década de los cincuenta, cuando se instalaron las colonias agrícolas con inmigrantes europeos, y en los años setenta, cuando el Programa de Reforma Agraria intentó desarrollar Áreas Rurales de Desarrollo Integral. Ambos proyectos nunca pudieron consolidarse por motivos estructurales y coyunturales (Rojas \& Pulido, 2009).

Estas propuestas que se recogen en el Plan Nacional de Desarrollo Regional 2001-2007 (República Bolivariana de Venezuela, 2001), no hacen más que retomar y reforzar con mayor énfasis la idea que atribuía a los desequilibrios 
territoriales la causalidad de las desigualdades sociales. Esta apreciación, tanto de los desequilibrios como de la excesiva concentración de la actividad económica y de población, ya aparecía en los diagnósticos que sustentaban los anteriores ${ }^{17}$ planes de la nación, incluso antes de la implementación y aplicación de las medidas neoliberales, hecho que ocurre con el segundo gobierno del presidente Carlos Andrés Pérez a inicios de los años 90 del pasado siglo XX.

Así, esta estrategia no solo reproduce los antiguos diagnósticos de las pasadas administraciones, sino que proyecta activar nuevos ejes de desarrollo territorial en función de enormes inversiones públicas en áreas poco pobladas, mientras se desatienden aquellas que más lo requieren por su importancia demográfica y económica. Ello revela la poca atención prestada a la historia territorial del poblamiento y a las fuerzas de la dinámica distributiva del ingreso petrolero, que seguramente continuarán atrayendo recursos y población hacia las áreas más dinámicas del territorio. Por otro lado, no se toman en cuenta experiencias anteriores de proyectos territoriales, todos con similar orientación: Programa

17 El IV Plan de la Nación (1970-74) formulaba una política de desconcentración económica y de descentralización administrativa. El V Plan (1976-80) se refiere a una dualidad espacial en Venezuela y a los desequilibrios interregionales y urbanos. EI VI Plan (1981-1985) insiste en la idea de los desequilibrios interregionales y propone estimular las áreas que el anterior calificaba de incentivos preferenciales. De esta manera, el nuevo plan (2001-2007) no hace más que ratificar los diagnósticos precedentes, atribuyéndole esta vez una causa (el neoliberalismo) que, a lo sumo, solo pudo haber reforzado las tendencias anteriores (Rojas, 2001). de la Conquista del Sur del Orinoco, Programa Integral de los Llanos Altos Occidentales, Proyecto Ciudad Sucre en la frontera con Colombia, Eje Fluvial Orinoco-Apure, entre otros.

Esta búsqueda por el equilibrio territorial se mantiene vigente para el segundo gobierno del presidente Chávez (2007-2012). Está presente tanto en el nuevo plan de desarrollo: Proyecto Nacional Simón Bolívar, Primer Plan Socialista 2007-2013 (República Bolivariana de Venezuela, 2007), como en la Nueva Geometría del Poder, uno de los cinco motores constituyentes definidos por su administración (Ley Habilitante, Reforma Constitucional, Explosión del Poder Comunal, Moral y Luces). Estos motores fueron anunciados el día de su juramentación (10 de diciembre, 2006), con el objeto de otorgarle el necesario marco legal al nuevo sistema político y económico que promovía. En particular, con la Nueva Geometría del Poder se planteaba:

¿Cómo lograr una relación simétrica o una ampliación del poder político, del poder económico, del poder social, del poder militar, a lo largo y ancho de todo el territorio? (...) Hay alcaldías o municipios que tienen una extensión de territorio gigantesca, hay otros que tienen poco territorio $\mathrm{y}$ están súper poblados, (...). Hay que comenzar por los pequeños detalles, por desmontar los privilegios las malas costumbres del estado liberal burgués (Ministerio 
del Poder Popular para la Infraestructura, 2007).

Es evidente que esta propuesta para ordenar el territorio nacional se sustenta en la ilusión de que su actual organización no ha respondido a las necesidades de la sociedad, sino a las del capital, por lo que se requiere revertir esta histórica ocupación a través de un modelo de desarrollo que tendría en la noción de 'equilibrio' su pivote fundamental. Esta manera de entender la organización histórica del territorio venezolano supondría darle atención prioritaria a las áreas menos pobladas del país, desfavoreciendo así al área centro norte, en la que viven más de ocho millones de personas y que incluye, además de Caracas, al principal aeropuerto internacional (Maiquetía) y al puerto natural de mayor capacidad del Caribe (Puerto Cabello), (Pulido \& Trinca, 2007).

La noción de cómo 'descentralizar y desconcentrar' el territorio, la materializa nuevamente el presidente Chávez al presentar su proyecto de reforma constitucional en agosto del 2007:

...el territorio y su organización político-territorial tienen un peso sumamente grande a la hora de pretender hacer cambios revolucionarios. Una revolución no puede serlo realmente si no enfoca el problema geográfico y de la distribución del poder político, económico, social, militar sobre su espacio;...la geografía... tiene un peso muy grande (...)" "...no podemos seguir picando a Vene- zuela en pedazos, aquí no podemos seguir aceptando o generando situaciones sobre las que se van formando caudillitos o caudillos, que pretenden ser presidenticos de republiquetas o republiquitas. Venezuela es una sola y una sola la unidad del territorio. (Negrilla y subrayado nuestro), (Estaba, 2008).

Aun cuando el proyecto de reforma constitucional fue rechazado en la consulta popular del 2 de diciembre de 2007, el Ejecutivo Nacional persiste en instrumentar la nueva geometría del poder, así como los otros 'motores', mediante leyes habilitantes u otros medios legislativos. En el proyecto de reforma se planteaba modificar el artículo 16 de la Constitución, de la siguiente manera:

El territorio nacional se conforma para los fines políticos-territoriales, y de acuerdo con la nueva geometría del poder, por un distrito federal en el cual tendrá su sede la Capital de la República, por los estados, las regiones marítimas, los territorios federales, los municipios federales y los distritos insulares. (...) Los estados se organizan en municipios.

La unidad política primaria de la organización territorial nacional será la ciudad, entendida ésta como todo asentamiento poblacional dentro del municipio, e integrada por áreas o extensiones geográficas denominadas comunas. Las comunas serán las 
células geo-humanas del territorio y estarán conformadas por las comunidades, cada una de las cuales constituirá el núcleo espacial básico e indivisible del Estado Socialista Venezolano, donde los ciudadanos y las ciudadanas comunes tendrán el poder para construir su propia geografía y su propia historia.

A partir de la comunidad y la comuna, el Poder Popular desarrollará formas de agregación comunitaria político-territorial, las cuales serán reguladas en la Ley, y que constituyan formas de Autogobierno y cualquier otra expresión de Democracia Directa.

La ciudad comunal se constituye cuando en la totalidad de su perímetro, se hayan establecido las comunidades organizadas, las comunas y los autogobiernos comunales, estando sujeta su creación a un referéndum popular que convocará el Presidente de la República en Consejo de Ministros.

El Presidente de la República, en Consejo de Ministros, previo acuerdo aprobado por la mayoría simple de los diputados y diputadas de la Asamblea Nacional, podrá crear mediante decreto, provincias federales, ciudades federales y distritos funcionales, así como cualquier otra entidad que establezca la Ley (....).

A pesar de no haber sido aprobada la reforma constitucional, el Ejecutivo
Nacional continúa en su empeño por el control territorial de las regiones, apoyado en el control político de la Asamblea Nacional, a espaldas del régimen federal descentralizado consagrado en el artículo 4 de la vigente Constitución Nacional. Así vemos como a mediados del 2008 se aprueba en la Asamblea Nacional, un conjunto de 26 decretos leyes introducidos por el presidente de la República, que recogen objetivos del proyecto de reforma constitucional. Dos ejemplos ilustran (Rojas \& Pulido, 2009) esta insistencia: a) el artículo 70 de la Ley Orgánica de la Administración Pública (República Bolivariana de Venezuela, 2008) dice: La Presidenta o Presidente de la República podrá designar autoridades regionales, las cuales tendrán por función la planificación, ejecución, seguimiento y control de las políticas, planes y proyectos de ordenación y desarrollo del territorio aprobados conforme a la planificación centralizada, así como, las demás atribuciones que les sean fijadas de conformidad con la Ley, asignándoles los recursos necesarios para el eficaz cumplimiento de su objeto; b) la Ley Orgánica de la Fuerza Armada Nacional Bolivariana (Asamblea Nacional, 2008) faculta al Presidente para crear Regiones Estratégicas de Defensa cuyos comandantes pueden asumir roles jerárquicos sobre gobernadores y alcaldes.

Sin pretender emitir juicios de valor con relación a cuán bueno o malo pueda ser el legado que nuestro reciente pasado petrolero nos dejó territorialmente hablando, no podemos negar que el país reclama políticas territoriales que tomen 
en cuenta la historia de su proceso de ocupación. Pero estas no deberían sustentarse en la idea del equilibrio, tal como lo hicieron los gobiernos del período democrático y lo continúa haciendo la administración del Gobierno del presidente Chávez. No se debería olvidar que la búsqueda de la equidad social, sin dejar de lado sus otros componentes, no tiene un correlato territorial.

\section{Conclusiones}

Al ser el territorio el nombre político del espacio, cualquier realidad nacional presenta una determinada organización que se corresponde con su proceso de formación, que los incluye a ambos (territorio usado). Sobre esta organización se interviene, desde el Estado, con la finalidad expresa de condicionar o influir sobre el desarrollo y el bienestar de sus habitantes. Es a este proceso ejecutivo que responde la denominada ordenación del territorio.

Si la organización espacial de un país cualquiera, muestra la historia de su territorio, mal se podría pensar que esa historia se construyó de manera no equitativa, o sin criterios de justicia social: no es equiparable la equidad social con la equidad territorial. La historia nos dice que la forma como los seres humanos, organizados socialmente, han tomado posesión de la base material que les da sustento, ha sido $-\mathrm{y}$ es- desigual. El uso diferencial que hacen de sus territorios reafirma que el proceso de ocupación no se realiza al margen de las intenciones que guían u orientan a esos grupos. Pero también dice que esas intenciones se materializan en objetos cuya existencia tiene que ver con las necesidades propias de cada momento histórico, los que, al acumularse de manera desigual en los territorios (Santos, 1996), le van incorporando valores diferenciales a los sistemas de objetos. Estos terminan, de manera progresiva, por imponer realidades que deben ser, necesariamente, consideradas a la hora de tomar decisiones que signifiquen alteraciones en los usos de esos territorios.

Por lo señalado, la confusa relación causal que se desprende entre diversidad territorial y desigualdad social, contenida en los variados planes de desarrollo que se han diseñado e instrumentado a lo largo de más de medio siglo en Venezuela, parte de un supuesto errado, pues los territorios, por definición, no son homogéneos; la diferencia es una de sus características constitutivas: "las cosas y los objetos naturales y culturales nunca se distribuyen uniformemente en el espacio" (Rojas \& Pulido, 2009, p. 93); la desigualdad social tiene que ver con la forma como se concretiza un sistema económico en el tiempo y el espacio, no con las diferencias propias de los territorios.

De esta manera, la "desigualdad" territorial no se acompaña necesariamente de la desigualdad social, así como tampoco equilibrio territorial significa justicia distributiva. Todo apunta a que se trata de una valoración respecto a la distribución de las actividades y de la población, que considera que hay muchas 
personas en algunos lugares y pocas en otros. Visto de esta manera, se definiría el desequilibrio con respecto a un modelo ideal, no explícito, según el cual se debería buscar una distribución de las actividades del hombre social que fuese más o menos igual en todo el territorio; cualquier distribución distinta se consideraría injusta, no equilibrada.

Tomar en cuenta la diversidad del espacio con miras a buscar metas sostenibles en lo económico, social y ambiental, no deja de lado el cuestionamiento de las desigualdades sociales y los fundamentalismos territoriales extremos. La diferencia de los territorios no está reñida, por tanto, con la equidad social que debe regir entre sus habitantes. Por último, se comparte con Rojas y Pulido (2009), que es la interdependencia de espacio y sociedad en el transcurso del tiempo la que da origen a una estructura territorial unificada, apropiada, heterogénea, generadora y recibidora de gran variedad de flujos. Es la razón por la cual se identifica al territorio como categoría socioespacial en el pensamiento geográfico contemporáneo.

La configuración territorial que presenta la Venezuela de nuestros días, se sustenta en este proceso que nos coloca delante de una sociedad que se localizó y concentró en aquellos lugares que le permitieron y permiten optimizar, en su etapa petrolera, su alta capacidad de importación; lugares que no por casualidad fueron los mismos del período agroexportador.

Más allá de la quimera del equilibrio territorial, Venezuela necesita, entre muchas cosas, volver la mirada hacia las regiones y las ciudades de mayor tamaño, con el objeto de acondicionarlas y equiparlas para asegurarle no solo una mejor calidad de vida a sus habitantes, sino también viabilizar sus economías y potenciar una mayor autonomía política y administrativa. Con esta afirmación no se sugiere que hay que desatender el campo o las áreas menos pobladas del país; por el contrario, lo que se señala es que deben preferirse las demandas de las regiones, las cuales se localizan, mayoritariamente en las ciudades, que se concentran, de manera importante, en la región centro-norte-costera, pero también en el resto del arco montañoso andino-costero. No es por azar que la población de la Venezuela de nuestros días, a diferencia de la que despertó con el siglo XX, sea fundamentalmente urbana y se concentre en los lugares y regiones donde siempre se ha localizado. Revertir esta realidad partiendo de que esto es un error, es desconocer la historia territorial de este país. 


\section{Literatura citada}

Baptista, A. (1985). Más allá del optimismo y del pesimismo: las transformaciones fundamentales del país. En: El caso Venezuela. Una ilusión de armonía. (pp. 20-41). Caracas: IESA, C.A.

Bosque, J., Díaz, C. \& Díaz, M. A. (2004). De la justica espacial a la justicia ambiental en la política de localización de instalaciones para la gestión de residuos en la comunidad de Madrid. Geográfica Digital, 1(2). Recuperado de http://hum.unne.edu.ar/investigación/geografia/igunne/index.htm

Chaves, L. F. (1992). Geografía humana de Venezuela. Mérida: Escuela de Geografía, Universidad de Los Andes.

Estaba, R. (1985). Formación de espacios regionales y urbanización en Venezuela: sus raíces históricas. Tesis no publicada. Escuela de Geografía, Universidad Central de Venezuela, Caracas.

Estaba, R. (2008). Venezuela: ¿Nueva geometría del poder? Ponencia presentada en la Conferencia mundial sobre desarrollo de ciudades, celebrada en Porto Alegre, Brasil.

Fournier, J. M. (2011). Existe-t-il une nouvelle géographie socialiste au Venezuela? Justice spatiale et justice sociale dans la révolution bolivarienne de Hugo Chavez. Cybergeo: European Journal of Geography, Politique, Culture, Représentations, article 518, mis en ligne le 25 février 2011, modifié le 02 mars 2011. Recuperado de http://cybergeo.revues.org/23477

Harvey, D. (1977). Urbanismo y desigualdad social. Madrid: Siglo XXI.

Harvey, D. (1992). Condição pós-moderna. São Paulo: Edições Loyola, p. 349. ( $1^{\text {a }}$ edição em ingles, 1990). The condition of posmodernity: an enquiry into the origins of cultural change. Oxford: Blackwell, UK.

Harvey, D. (1996). Justice, nature and the geography of difference. Oxford: Blackwell, UK.

Interficto. (2010, 29 de octubre). ¿Qué es la justicia social? Recuperado de http:// www.articulo.org/articulo/25481/que_es_la_justicia_social.html

Lefebvre, H. (1974). La production de l'espace. Paris: Anthropos.

Link, L. F. (2011). Seeking spatial justice. Revista EURE, 37(111), 173-177.

Mapas de relieve de Venezuela (2012). Recuperado de: http://www.a-venezuela. $\mathrm{com} / \mathrm{mapas} / \mathrm{map} / \mathrm{html} /$ relieve.html 
Ministerio para el Poder Popular para la Infraestructura. (2007). 5 Motores a máxima revolución... jrumbo al socialismo! ( $1^{\mathrm{a}}$ ed.). Caracas: Gobierno Bolivariano de Venezuela.

Moraes, A. C. \& Messias da Costa, W. (1987). A valoração do espaço. São Paulo: Hucitec.

Peet, R. (1977). Radical geography: Alternative viewpoints on contemporary social issues. London: Methuen.

Pulido, N. \& Trinca, D. (2007). Remedios urbanos: por encima del debate teórico. Scripta Nova, Revista electrónica de Geografía y Ciencias Sociales, XI (245). [En línea], Universidad de Barcelona, España. Recuperado de http:// www.ub.edu/geocrit/sn/sn-24506.htm.

Rawls, J. (1971). A theory of justice. Cambridge: Harvard University Press.

República Bolivariana de Venezuela. (2001). Plan Nacional de Desarrollo Regional 2001-2007. Caracas: Ministerio de Planificación y Desarrollo. Despacho del Viceministro de Planificación y Desarrollo Regional.

República Bolivariana de Venezuela. (2007). Proyecto Nacional Simón Bolívar. Primer Plan Socialista (PPS). Desarrollo Económico y Social de la Nación 2007-2013. Caracas: Presidencia.

República Bolivariana de Venezuela. (2008, 31 de julio). Ley Orgánica de la Administración Pública. Caracas: Imprenta Nacional, Gaceta Oficial N 5.890.

República de Venezuela. (1983, 11 de agosto). Ley Orgánica para la Ordenación del Territorio. Caracas: Gaceta Oficial $N^{\circ} 3.238$ Extraordinario.

Reynaud, A. (1981). Société, space et justice. Inégalités regionales et justice sociospatiale. Paris: Presses Universitaires de France.

Rojas, A. (2001). La dinámica políticoterritorial de Venezuela, los planes de ordenamiento y el ingreso petrolero. Revista Geográfica Venezolana, 42 (2), 183-201.

Rojas, J. J. \& Pulido, N. (2009). Estrategias territoriales recientes en Venezuela: ¿reordenación viable de los sistemas territoriales o ensayos de laboratorio? Revista EURE, 35(104), 77-100.

Rojas, J. J. \& Gómez, E. (2010). Tiempos del pensamiento geográfico. Mérida: Arquidiócesis de Mérida. Archivo Arquidiocesano de Mérida. Serie. Estudio, 9. Escuela de Geografía, Universidad de Los Andes, 181 p. 
Salas-Bourgoin, M. A., Pulido, N., Rojas, J. J. \& Páez, G. (2011). Desarrollo territorial en Venezuela: una mirada desde lo actual. (CD-Rom). En: A. Olmos, A. Massiris, J. Farinós, M.V. Santana \& E. M. Rosales (comp.). Dinámicas territoriales, políticas de desarrollo territorial sostenible y nueva gobernanza territorial en el espacio Iberoamericano. Conceptos, métodos y tendencias. Toluca, México: Universidad Autónoma del Estado de México, Facultad de Geografía; Universidad Pedagógica y Tecnológica de Colombia; Universidad de Valencia; Red Nacional de Instituciones Universitarias Geográficas de México (RENIG) 32.

Santos, M. (1975). L'espace partage: les deux circuits de l'economie urbaine de pays sousdéveloppés. Paris: Librarires Techniques.

Santos, M. (1996). A natureza do espaço. Técnica e tempo. Razão e Emoção. São Paulo: Hucitec.

Slater, D. (1973). Geography and underdevelopment. Antipode, 5, 21-33.

Smith, N. (1988). Desenvolvimento desigual. Rio de Janeiro: Bertrand Brasil, S.A. ( $1^{\text {a }}$ ed. en inglês, 1984).

Smith, D. (1994). Geography and social justice. Oxford: Blackwell.

Soja, E. (1993). Geografias pós-modernas. A reafirmação do espaço na teoria social crítica. Rio de Janeiro: Jorge Zahar.

Soja, E. (2010, 13 de junio). Entrevista a Edward Soja. Parte 3/11. Recuperado de http://lageografiaweb.blogspot.com/2010/06/entrevista-edward-sojaparte-11.html

Trinca, D. (2005). A Venezuela e os desafios territoriais do presente. Em: Continente em Chamas. Globalização e Território na América Latina. Rio de Janeiro: Civilização Brasileira.

Trinca, D. (2008). Venezuela, petróleo y territorio. Boletín de la Real Sociedad Geográfica, CXLIV, 151-170. Madrid.

Venezuela Analítica. (2001). Programa de gobierno de la administración del Presidente Hugo Chávez Frías. Recuperado de http://www.analitica.com/bitblioteca/

Recepción: 14 de julio de 2012

Evaluación: 10 de octubre de 2012

Aprobación: 15 de febrero de 2013 